\title{
Adaptive Robust Control of Servo Mechanisms With Compensation for Nonlinearly Parameterized Dynamic Friction
}

\author{
Zhiping Li, Student Member, IEEE, Jie Chen, Member, IEEE, Guozhu Zhang, and Minggang Gan
}

\begin{abstract}
In this brief, an adaptive robust control (ARC) scheme with compensation for nonlinearly parameterized dynamic friction is proposed. Both parametric uncertainties and external disturbances are considered in this method. Our method takes advantage of a Lipschitzian property with respect to the parameters of nonlinearly parameterized model in the ARC design. The outcome is that the number of parameters to be updated in the ARC is equal to the number of unknown parameters in the plant, and thus the resulting control algorithm is convenient to be implemented. We have proved theoretically that the proposed method can not only guarantee desired transient performance for the system, but also make the magnitude of steady-state tracking error to be arbitrarily small in the presence of parametric uncertainties only. Experimental results are given to demonstrate the effectiveness of the proposed ARC scheme.
\end{abstract}

Index Terms - Adaptive robust control (ARC), friction compensation, servo mechanism.

\section{INTRODUCTION}

I N SERVO mechanisms, friction may cause many undesired phenomena such as large tracking errors, limit cycles, and stick-slip motion. Accordingly, it is important to compensate for the effects of friction, when high performance is needed for servo mechanisms. Many methods have been proposed to solve the friction compensation problem [1], [2]. Some control schemes (e.g., in [3], [4]) are based on an accurate offline friction estimation. The main drawback of this kind of methods is that, their design procedures need accurate models of friction, which are difficult to acquire. To overcome this problem, adaptive friction compensation techniques based on different friction models have been proposed in the literature [5]-[7]. In most of these results, friction is modeled as a static map between velocity and friction. However, in applications with high precision positioning and with low velocity tracking, friction compensation based on static models is not always satisfactory.

Several behaviors of friction, such as presliding displacement, hysteresis, friction lag, and etc., cannot be represented by the static models. To capture these effects, researchers have proposed a number of dynamic friction models [8]. For example, Dahl [9] proposed a dynamic model to capture the spring-like behavior during stiction. In [10], a dynamic friction

Manuscript received April 07, 2011; revised September 18, 2011; accepted October 06, 2011. Manuscript received in final form October 08, 2011. Date of publication December 06, 2011; date of current version December 14, 2012. This work was supported by the National Science Fund for Distinguished Young Scholars (60925011). Recommended by Associate Editor F. Caccavale.

$\mathrm{Z}$. Li is with the Beijing Institute of Control Engineering, Beijing 100080, China (e-mail: lizplst@gmail.com).

J. Chen, G. Zhang, and M. Gan are with the School of Automation, Beijing Institute of Technology, Beijing 100081, China.

Digital Object Identifier 10.1109/TCST.2011.2171966 model called LuGre model is proposed. This model captures various friction effects such as the Stribeck effect, hysteresis, presliding displacement and varying break-away force.

Based on the LuGre model, several model-based controllers have been developed. In [10], a model-based controller with a friction state observer was proposed, under the assumption that the system parameters are exactly known. However, it is usually difficult to capture the nonlinear features of friction exactly, since every physical system is subject to certain degrees of model uncertainties. To account for the uncertainties in the LuGre model, some adaptive controllers have been presented. Two globally stable model-based adaptive friction compensation schemes were proposed in [11] to address parameter changes associated with normal force variation or temperature variation. In [12], an adaptive controller utilizing a nonlinear observer/filter structure was proposed to handle non-uniform variations in the friction force by assuming independent coefficient change as temperature varies. In [13], an adaptive controller is developed by introducing a nonlinear observer, which is utilized to estimate the unmeasurable friction state. In [14], the neural network is used to parameterize the unknown dynamic friction bounding function, and then an adaptive neural network controller is developed. However, these adaptive controllers suffer from two main drawbacks - unknown transient performance and possible non-robustness to disturbances.

To overcome these drawbacks, a dynamic friction compensation strategy is proposed in [15] by utilizing the idea of adaptive robust control (ARC) [16], [17], where the robust control term is used to guarantee desired transient response, and the adaptive control term is used to achieve favorable steady-state tracking accuracy. In this method, the parameters related to the Stribeck effect are assumed to be known. With these known parameters, a dual-observer is constructed to estimate the unknown friction states. A similar result is proposed in [18]. However, different from the method in [15], a modified LuGre model is adopted in [18], and thus the digital implementation problems of the LuGre-model-based dynamic friction compensation can be avoided. However, the above adaptive robust controllers are based on the assumption of known Stribeck curves, which is not easy to obtain.

In this brief, a novel ARC-based dynamic friction compensation strategy is proposed. This method does not need previously known Stribeck-effect-related parameters in its design procedure. Thus, it is more convenient for engineering application than the adaptive robust controllers proposed in [15] and [18]. Unlike the adaptive neural network friction compensator proposed in [14], the friction compensator is synthesized directly according to the nonlinearly parameterized model in our method. Hence, the number of parameters to be estimated is equal to the number of unknown parameters of the plant, which 
is much less than the parameter number of the adaptive neural network controller in [14]. Motivated by the recently proposed method in [19] and [20], our method takes advantage of a Lipschitzian property with respect to the parameters of nonlinearly parameterized model in the ARC design. Unlike [19] and [20], where the friction compensation is based on Stribeck model and only the parametric uncertainty is considered, this brief focuses on the adaptive robust control with friction compensation based on LuGre model for a DC servomechanism subjected to parametric and nonlinear uncertainties.

This brief is organized as follows. The problem description is presented in Section II. The design procedure of the proposed ARC is provided in Section III. The stability proof and performance analysis are given in Section IV. Experimental results are described and analyzed in Section $\mathrm{V}$ and conclusions are drawn in Section VI.

\section{Dynamic Models AND Problem Formulations}

\section{A. Dynamic Model of Servo Mechanisms}

By ignoring the current dynamics, the mechanical and electrical dynamics of a current-controlled DC servo mechanism can be described as [21]

$$
\begin{aligned}
J \ddot{q} & =T_{m}-T_{f}-T_{l}-T_{\mathrm{dis}} \\
T_{m} & =K_{I} u_{I}
\end{aligned}
$$

where $q$ is the angular position; $u_{I}$ is the current input command; $T_{m}, T_{f}, T_{l}$, and $T_{\mathrm{dis}}$ represent the torques imposed on the motor shaft: generated torque, friction torque, load torque, and disturbance torque, respectively; $J$ represents the total motor and load inertia; $K_{I}$ denotes the electrical-mechanical energy conversion constant. Using the dynamic friction model proposed in [10], the dynamic friction torque $T_{f}$ can be described by

$$
\begin{aligned}
T_{f} & =\sigma_{0} z+\sigma_{1} \dot{z}+\sigma_{2} \dot{q} \\
\dot{z} & =\dot{q}-\sigma_{0} \frac{|\dot{q}|}{g(\dot{q})} z
\end{aligned}
$$

where $z$ represents the unmeasured internal friction state; $\sigma_{0}$, $\sigma_{1}$, and $\sigma_{2}$ are positive parameters that can be physically explained as the stiffness of bristles, damping coefficient associated with $\dot{z}$, and viscous coefficient. The function $g(\dot{q})$ is utilized to describe the Stribeck effect [10] and given as follows:

$$
g(\dot{q})=T_{c}+\left(T_{s}-T_{c}\right) e^{-\left(\dot{q} / \omega_{s}\right)^{2}}
$$

where $\omega_{s}$ is the Stribeck velocity; $T_{c}$ and $T_{s}$ represent the levels of Coulomb friction and stiction, respectively. In general, $0<$ $T_{c} \leq T_{s}$, thus, (5) implies that $T_{c} \leq g(\dot{q}) \leq T_{s}$. The friction model given by (3)-(5) reflects the dynamic effects caused by the deflection of bristles, which are used to model the asperities between two contacting surfaces. It is shown in [11] and [22] that the above friction model has the following finite bristle deflection property.
Property 1: Under the assumption that $0<z_{\min } \leq$ $\left(g(\dot{q}) / \sigma_{0}\right) \leq z_{\max }$, we have, if $|z(0)| \leq z_{\max }$, then $|z(t)| \leq z_{\max }, \forall t \geq 0$.

This property will be used in the subsequent controller design. Here, all the parameters of the plant (1)-(5) are assumed to be unknown. Thus, it can be concluded that the plant studied in this brief is subject to dynamic friction, parametric uncertainties and external disturbance. The angular position $q$ is regarded as the system output. Defining the angular position and angular velocity as the state variables, i.e., $\left[x_{1}, x_{2}\right]^{T}=[q, \dot{q}]^{T}, T_{n}$ as the mean value (direct offset) of $T_{l}+T_{\text {dis }}$ and $d$ as the disturbance term, i.e., $d=T_{n}-T_{l}-T_{\text {dis }}$, from (1)-(5), the entire system can be expressed in the state space form as

$$
\left\{\begin{array}{l}
\dot{x}_{1}=x_{2} \\
\dot{x}_{2}=\alpha_{1} u_{I}-\alpha_{2} x_{2}-\alpha_{3}-T_{f n}\left(x_{2}, \sigma_{0} z, \beta\right)+\Delta \\
\dot{z}=x_{2}-\sigma_{0} \frac{\left|x_{2}\right|}{g\left(x_{2}\right)} z \\
y=x_{1} \\
T_{f n}\left(x_{2}, \sigma_{0} z, \beta\right)=\sigma_{0} z\left[\beta_{1}\left(1-\frac{\beta_{2}\left|x_{2}\right|}{1+\left(\beta_{3}-1\right) e^{-\beta_{4} x_{2}^{2}}}\right)\right]
\end{array}\right.
$$

where $\Delta=d / J$ denotes the lumped disturbance; $\alpha:=$ $\left[\alpha_{1}, \alpha_{2}, \alpha_{3}\right]^{T}=\left[K_{I} / J,\left(\sigma_{1}+\sigma_{2}\right) / J, T_{n} / J\right]^{T}$ is the parameter vector consisting of the linearly parameterized coefficients; $\beta:=\left[\beta_{1}, \beta_{2}, \beta_{3}, \beta_{4}\right]^{T}=\left[1 / J, \sigma_{1} / T_{c}, T_{s} / T_{c}, 1 / \omega_{s}^{2}\right]^{T}$ is the parameter vector consisting of the nonlinearly parameterized coefficients.

\section{B. Assumptions and Problem Formulations}

For simplicity, the following notations will be used: $\bullet_{i}$ for the $i$ th component of the vector $\bullet$, $\hat{\bullet}$ for the estimate of $\bullet, \bullet$ min for the minimum value of $\bullet$, and $\bullet_{\max }$ for the maximum value of -. The operation $\leq$ for two vectors is performed in terms of the corresponding elements of the vectors.

In this brief, we assume that the uncertain parameters are in certain known intervals, as shown in Assumptions $1 \sim 3$. In addition, Assumption 4 is made for the desired motion trajectory $x_{1 d}(t)$.

Assumption 1: $\alpha_{i} \in\left[\alpha_{i \min }, \alpha_{i \max }\right], \beta_{j} \in\left[\beta_{j \min }, \beta_{j \max }\right]$ $(i=1, \ldots, 3, j=1, \ldots, 4)$, where $\alpha_{i \min }, \alpha_{i \max }, \beta_{j \min }$, and $\beta_{j \text { max }}$ are previously known positive numbers. In addition, it is assumed that $\alpha_{1 \min }>0$ and $\beta_{3 \min } \geq 1$, which is coherent with the fact that $J>0$ and $T_{s} \geq T_{c}$.

Assumption 2: The stiction is upper bounded, i.e., $T_{s} \leq$ $T_{\max }$, where $T_{\max }$ is known.

Assumption 3: The disturbance $\Delta$ is bounded, i.e., $|\Delta| \leq \delta$, where $\delta$ is known.

Assumption 4: The desired trajectory $x_{1 d}$ is continuous with first derivative $\dot{x}_{1 d}$ and second-order derivative $\ddot{x}_{1 d}$ available.

Consider model (6) which has dynamic friction nonlinearity, parametric uncertainties and disturbance. The control problem of this brief can be stated as follows. Given the desired motion trajectory $x_{1 d}(t)$, the objective is to synthesize a control input $u_{I}$ such that the system output $y=x_{1}$ tracks $x_{1 d}(t)$ as closely as possible in spite of dynamic friction and various model uncertainties. 


\section{AdAPTIVE RoBUSt CONTROL}

In this section, we are going to detail the adaptive robust control design for the plant (6).

\section{A. Discontinuous Control Law}

The control law design follows the ARC synthesis procedure proposed in [16], [17] and [25]. First, define a tracking-errorindex-like variable [26] $e_{2}$ as

$$
e_{2}=\dot{e}_{1}+k_{p} e_{1}=x_{2}-x_{2 e q}, \quad x_{2 e q}:=\dot{x}_{1 d}-k_{p} e_{1}
$$

where $e_{1}=x_{1}-x_{1 d}(t)$ is the output tracking error, and $k_{p}$ is any positive feedback gain. Since $G_{s}(s)=e_{1}(s) / e_{2}(s)=$ $1 /\left(s+k_{p}\right)$ is a stable transfer function, if $e_{2}$ is small or converges to zero exponentially, then the output tracking error $e_{1}$ will be small or converge to zero exponentially. Differentiating (7), from (6), we obtain

$$
\begin{aligned}
\dot{e}_{2} & =\dot{x}_{2}-\dot{x}_{2 e q} \\
& =\alpha_{1} u_{I}-\alpha_{2} x_{2}-\alpha_{3}-T_{f n}\left(x_{2}, \sigma_{0} z, \beta\right)+\Delta-\dot{x}_{2 e q}
\end{aligned}
$$

where $\dot{x}_{2 e q}:=\ddot{x}_{1 d}-k_{p} \dot{e}_{1}$. Define a positive definite function $V$ as $V\left(e_{2}\right)=(1 / 2) e_{2}^{2}$. Then, from (8), the derivative of $V$ can be derived as

$\dot{V}=e_{2}\left(\alpha_{1} u_{I}-\alpha_{2} x_{2}-\alpha_{3}+\Delta-\dot{x}_{2 e q}\right)-e_{2} T_{f n}\left(x_{2}, \sigma_{0} z, \beta\right)$.

From the right-hand side of (9), we observe that a nonlinearly parameterized term $e_{2} T_{f n}\left(x_{2}, \sigma_{0} z, \beta\right)$ influences the derivative of $V$.

Viewing Property 1 and Assumption 2, we obtain the following result.

Lemma 1: Under Assumption 2, if $\left|\sigma_{0} z(0)\right| \leq T_{\max }$, then $\left|\sigma_{0} z(t)\right| \leq T_{\max }, \forall t \geq 0$.

Proof: Noting that $0<T_{c} \leq g\left(x_{2}\right) \leq T_{s}$, from Assumption 2, we get $g\left(x_{2}\right) / \sigma_{0} \leq T_{\max } / \sigma_{0}$. Combining with Property 1 , it can be concluded that $|z(t)| \leq T_{\max } / \sigma_{0}, \forall t \geq 0$, if $|z(0)| \leq T_{\max } / \sigma_{0}$, which yields the result of Lemma 1 .

From Lemma 1, one has

$$
\begin{aligned}
& -e_{2} T_{f n}\left(x_{2}, \sigma_{0} z, \beta\right) \\
& \quad \leq e_{2} \operatorname{sgn}\left(e_{2}\right) T_{\max } \beta_{1}\left[1+\frac{\beta_{2}\left|x_{2}\right|}{1+\left(\beta_{3}-1\right) e^{-\beta_{4} x_{2}^{2}}}\right] .
\end{aligned}
$$

Define a nonlinearly parameterized function $f\left(x_{2}, \beta\right)$ as

$$
f\left(x_{2}, \beta\right)=\beta_{1}\left[1+\frac{\beta_{2}\left|x_{2}\right|}{1+\left(\beta_{3}-1\right) e^{-\beta_{4} x_{2}^{2}}}\right] .
$$

To find the bound of $f\left(x_{2}, \beta\right)$, the definition of Lipschitzian condition is recalled first.

Definition 1: The function $f\left(x_{2}, \beta\right)$ is said to be locally Lipschitzian in $\beta$ if there exist continuous functions $L_{i}\left(x_{2}\right), i=$ $1, \ldots, 4$ such that the following inequality holds:

$$
\left|f\left(x_{2}, \beta^{\prime}\right)-f\left(x_{2}, \beta^{\prime \prime}\right)\right| \leq \sum_{i=1}^{4} L_{i}\left(x_{2}\right)\left|\beta_{i}^{\prime}-\beta_{i}^{\prime \prime}\right| 1
$$

$$
\forall \beta^{\prime}, \beta^{\prime \prime} \in \Omega_{\beta}:=\left\{\beta \mid \beta_{i \min } \leq \beta_{i} \leq \beta_{i \max }, i=1, \ldots, 4\right\}
$$

where $\beta^{\prime}=\left[\beta_{1}^{\prime}, \beta_{2}^{\prime}, \beta_{3}^{\prime}, \beta_{4}^{\prime}\right]^{T}, \beta^{\prime \prime}=\left[\beta_{1}^{\prime \prime}, \beta_{2}^{\prime \prime}, \beta_{3}^{\prime \prime}, \beta_{4}^{\prime \prime}\right]^{T}$.

Lemma 2: The function $f\left(x_{2}, \beta\right)$ is Lipschitzian in $\beta$ if $\partial f\left(x_{2}, \beta\right) / \partial \beta$ exists and is continuous in $R \times \Omega_{\beta}$, furthermore, the continuous functions $L_{i}\left(x_{2}\right), i=1, \ldots, 4$ in (12) can be chosen as any continuous function satisfying $L_{i}\left(x_{2}\right) \geq \max _{\beta \in \Omega_{\beta}}\left|\partial f\left(x_{2}, \beta\right) / \partial \beta_{i}\right|$.

Proof: See Appendix A.

Then, from Lemma 2, we know that $f\left(x_{2}, \beta\right)$ given by (11) is locally Lipschitzian in $\beta$, because $\partial f\left(x_{2}, \beta\right) / \partial \beta$ exists and is continuous in $R \times \Omega_{\beta}$. Combining Lemma 2 with (11), and noting Assumption 1, it is easy to check that one choice of $L_{i}\left(x_{2}\right), i=1, \ldots, 4$ is

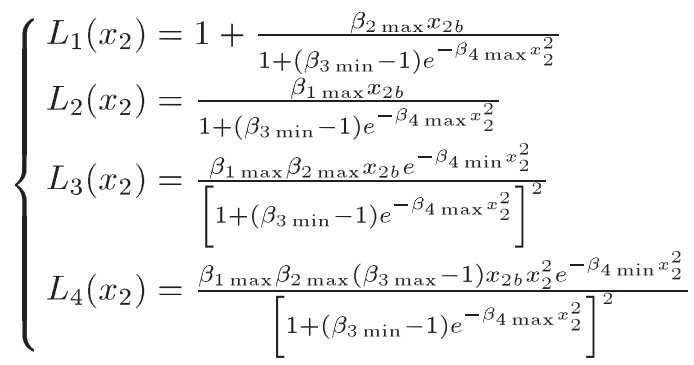

where $x_{2 b}=(1 / 4 b) x_{2}^{2}+b$ with $b>0$. In view of (10)-(13), it follows that

$$
\begin{aligned}
- & e_{2} T_{f n}\left(x_{2}, \sigma_{0} z, \beta\right) \\
\leq & e_{2} \operatorname{sgn}\left(e_{2}\right) T_{\max } L^{T}\left(x_{2}\right) \gamma-e_{2} T_{f n}\left(x_{2}, \sigma_{0} z, \beta_{\min }\right) \\
\leq & e_{2} \operatorname{sgn}\left(e_{2}\right) T_{\max } L^{T}\left(x_{2}\right) \gamma \\
& +e_{2} \operatorname{sgn}\left(e_{2}\right) T_{\max } f\left(x_{2}, \beta_{\min }\right)
\end{aligned}
$$

where $L\left(x_{2}\right):=\left[L_{1}\left(x_{2}\right), L_{2}\left(x_{2}\right), L_{3}\left(x_{2}\right), L_{4}\left(x_{2}\right)\right]^{T}$ and $\gamma$ is defined as

$$
\gamma=\beta-\beta_{\min }
$$

According to Assumption 1, we know that $\gamma_{\min } \leq \gamma \leq \gamma_{\max }$, where $\gamma_{\min }=0$ and $\gamma_{\max }=\beta_{\max }-\beta_{\min }$. For the convenience of deduction, we define the parameter vector $\theta$ as $\theta:=\left[\alpha^{T}, \gamma^{T}\right]^{T} \in R^{7}$. Then, it is easy to check that the upper and lower bounds of $\theta$ are $\theta_{\min }=\left[\alpha_{\min }^{T}, \gamma_{\min }^{T}\right]^{T}$ and $\theta_{\max }=\left[\alpha_{\max }^{T}, \gamma_{\max }^{T}\right]^{T}$.

Viewing (9), (14), and (15), we obtain

$$
\begin{aligned}
\dot{V} \leq e_{2}\left[\alpha_{1} u_{I}\right. & -\alpha_{2} x_{2}-\alpha_{3}+\operatorname{sgn}\left(e_{2}\right) T_{\max } L^{T}\left(x_{2}\right) \gamma \\
& \left.-\dot{x}_{2 e q}+\operatorname{sgn}\left(e_{2}\right) T_{\max } f\left(x_{2}, \beta_{\min }\right)+\Delta\right] .
\end{aligned}
$$

According to (16), the control law can be synthesized by using the ARC approach. The control input $u_{I}$ is designed as

$$
\left\{\begin{array}{c}
u_{I}=u_{a}+u_{s} \\
u_{a}=\frac{1}{\hat{\alpha}_{1}}\left[\hat{\alpha}_{2} x_{2}+\hat{\alpha}_{3}-\operatorname{sgn}\left(e_{2}\right) T_{\max } L^{T}\left(x_{2}\right) \hat{\gamma}+\dot{x}_{2 e q}\right. \\
\left.\quad-\operatorname{sgn}\left(e_{2}\right) T_{\max } f\left(x_{2}, \beta_{\min }\right)\right] \\
u_{s}=u_{s 1}+u_{s 2}, u_{s 1}=-\frac{k_{s 1}}{\alpha_{1 \min }} e_{2}
\end{array}\right.
$$


where $\hat{\alpha}_{i}$ is the estimate of $\alpha_{i}, i=1, \ldots, 4 ; \hat{\gamma}$ is the estimate of $\gamma ; k_{s 1}>0$ is a controller parameter to be chosen; $u_{a}$ is an adaptive control term; $u_{s}$ is a robust control term consisting of two parts, i.e., $u_{s 1}$ and $u_{s 2}$. The robust control function $u_{s 2}$ should be selected to satisfy

$$
\begin{aligned}
e_{2} u_{s 2} & \leq 0 \\
e_{2}\left[\alpha_{1} u_{s 2}-\varphi_{0}^{T} \tilde{\theta}+\Delta\right] & \leq \varepsilon_{0}
\end{aligned}
$$

where $\varphi_{0}^{T}=\left[u_{a},-x_{2},-1, \operatorname{sgn}\left(e_{2}\right) T_{\max } L^{T}\left(x_{2}\right)\right]^{T}$ and $\varepsilon_{0}>0$ is a design parameter. The parameter is updated by

$$
\dot{\hat{\theta}}=\operatorname{Proj}_{\hat{\theta}}(\Gamma \tau), \quad \Gamma>0
$$

where $\operatorname{Proj}(\bullet)$ represents the projection operation whose definition can be seen in [27], the positive definite matrix $\Gamma$ represents the adaptation rate, the adaptation function $\tau$ is synthesized as $\tau=\varphi_{0} e_{2}$. Since $\alpha_{1} \geq \alpha_{1 \text { min }}>0$ (as is indicated in Assumption 1) and the projection operator is used in the adaptation law to guarantee $\theta_{\text {min }} \leq \hat{\theta} \leq \theta_{\max }$, we know that there must be certain robust control function $u_{s 2}$ satisfying (18) and (19). There are many ways to design $u_{s 2}$. One alternative is to let

$$
u_{s 2}=-\frac{h_{0}}{2 \alpha_{1 \min } \varepsilon_{0}} e_{2}
$$

where $h_{0}$ is any function or constant satisfying $h_{0} \geq$ $\left\|\varphi_{0}\right\|^{2}\left\|\theta_{\max }-\theta_{\min }\right\|^{2}+\delta^{2}$. Readers can refer to [16] and [24] for other choices of $u_{s 2}$.

Lemma 3: Suppose that the control law (17) and the adaptation law (20) are applied to control the plant (6), which satisfies Assumptions $1 \sim 4$. Then, no matter what the adaptation function $\tau$ is, the controller guarantees that: The closed-loop system is globally stable, and the positive definite function $V$ defined by $V=(1 / 2) e_{2}^{2}$ is bounded above by

$$
V \leq \exp (-\lambda t) V(0)+\frac{\varepsilon_{0}}{\lambda}(1-\exp (-\lambda t))
$$

where $\lambda=2 k_{s 1}$.

Proof: Substituting (17) into (16), we have

$$
\begin{aligned}
& \dot{V} \leq-k_{s 1} e_{2}^{2} \\
&+e_{2}\left[\alpha_{1}\left(u_{a}+u_{s 2}\right)-\alpha_{2} x_{2}-\alpha_{3}-\dot{x}_{2 e q}\right. \\
&\left.+\operatorname{sgn}\left(e_{2}\right) T_{\max }\left(L^{T}\left(x_{2}\right) \gamma+f\left(x_{2}, \beta_{\min }\right)\right)+\Delta\right] \\
&=-k_{s 1} e_{2}^{2}+e_{2}\left[\alpha_{1} u_{s 2}-\tilde{\alpha}_{1} u_{a}+\tilde{\alpha}_{2} x_{2}+\tilde{\alpha}_{3}\right. \\
&\left.\quad-\operatorname{sgn}\left(e_{2}\right) T_{\max } L^{T}\left(x_{2}\right) \tilde{\gamma}+\Delta\right]
\end{aligned}
$$

Combining with (19), it follows that

$$
\dot{V} \leq-\lambda V+\varepsilon_{0}
$$

which implies (21).

Remark 1: Since $V=(1 / 2) e_{2}^{2}$ is nonincreasing and bounded from below by zero, according to the inequality (21), one has

$$
\left(\frac{1}{2} e_{2}^{2}(t)\right) \leq-\frac{1}{2} \exp (-\lambda t)\left|e_{2}(0)\right|^{2}+\frac{\varepsilon_{0}}{\lambda}(1-\exp (-\lambda t)) .
$$

It indicates that the tracking error $e_{2}(t)$ will exponentially converge to a bounded value $\varepsilon_{0} / \lambda$. There is no overshoot and the convergent rate can be prescribed by tuning parameter $\lambda$

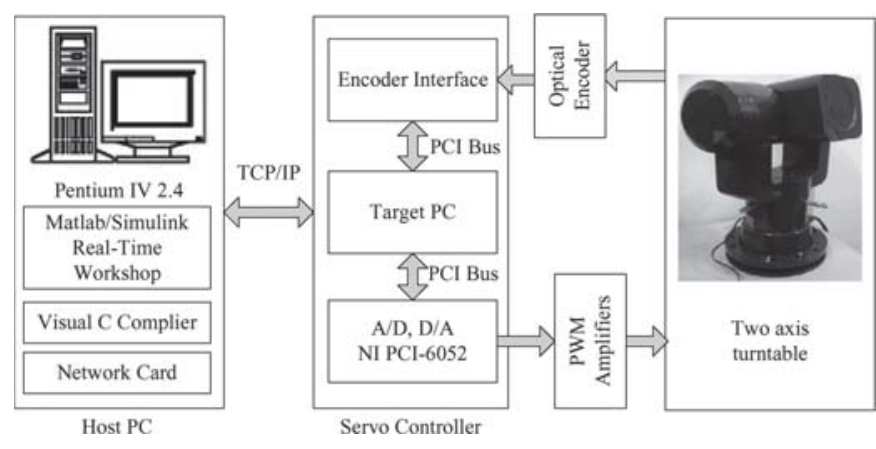

Fig. 1. Structure of the two-axis servo system.

which is determined by control gain. Moreover, in order to obtain better final tracking accuracy, smaller $\varepsilon_{0}$ or larger $\lambda$ is required which can be realized only by choosing large robust control gain when the parameter adaptation is closed. However, too large gain will lead the controller to be saturated. Thus, activating the adaptation mechanism is very helpful to reduce the conservatism of the controller.

Remark 2: Lemma 3 shows that the discontinuous control law can not only guarantee the closed-loop system to be stable, but also make the tracking error converge to a small prescribed region. However, due to the discontinuity at $e_{2}=0$ caused by the term $\operatorname{sgn}\left(e_{2}\right)$, the closed-loop system controlled by the control law (17) may suffer from chattering, which is not desired in the application. To solve this problem, in the next subsection, we will do some modification to the above control law design so that a continuous control action can be obtained.

\section{B. Continuous Control Law}

A continuous control law can be derived by modifying the tracking-error-index-like variable. Here, we introduce a new variable $e_{\varepsilon}$ as follows [19]:

$$
e_{\varepsilon}=e_{2}-\frac{1}{\sqrt{3}} c\left(e_{2}\right)
$$

where

$$
c\left(e_{2}\right)=\left\{\begin{array}{l}
d_{1}+\sqrt{d_{2}^{2}-\left(e_{2}-\varepsilon\right)^{2}}, \frac{\sqrt{3}-1}{2} \varepsilon \leq e_{2} \leq \varepsilon \\
\sqrt{3} e_{2}, \quad\left|e_{2}\right| \leq \frac{\sqrt{3}-1}{2} \varepsilon \\
-d_{1}-\sqrt{d_{2}^{2}-\left(e_{2}+\varepsilon\right)^{2}},-\varepsilon \leq e_{2} \leq \frac{1-\sqrt{3}}{2} \varepsilon \\
\varepsilon \operatorname{sgn}\left(e_{2}\right), \quad\left|e_{2}\right|>\varepsilon
\end{array}\right.
$$

with $d_{1}=(2-\sqrt{3}) \varepsilon, d_{2}=(\sqrt{3}-1) \varepsilon$, for $\varepsilon>0$. It has been indicated in [19] that $e_{\varepsilon}$ is a continuously differentiable function of $e_{2}$ (see Fig. 1). From (25), $e_{\varepsilon} / e_{2}$ can be derived as

$$
\frac{\partial e_{\varepsilon}}{\partial e_{2}}= \begin{cases}1+\frac{e_{2}-\varepsilon}{\sqrt{3\left[d_{2}^{2}-\left(e_{2}-\varepsilon\right)^{2}\right]},} & \frac{\sqrt{3}-1}{2} \varepsilon \leq e_{2} \leq \varepsilon \\ 0, & \left|e_{2}\right| \leq \frac{\sqrt{3}-1}{2} \varepsilon \\ 1-\frac{e_{2}+\varepsilon}{\sqrt{3\left[d_{2}^{2}-\left(e_{2}+\varepsilon\right)^{2}\right]},} & -\varepsilon \leq e_{2} \leq \frac{1-\sqrt{3}}{2} \varepsilon \\ 1, & \left|e_{2}\right|>\varepsilon .\end{cases}
$$

Define a positive definite function $V_{\varepsilon}$ as $V_{\varepsilon}\left(e_{\varepsilon}\right)=(1 / 2) e_{\varepsilon}^{2}$. Then, from (8), it follows that

$$
\begin{aligned}
\dot{V}_{\varepsilon}=e_{\varepsilon} \frac{\partial e_{\varepsilon}}{\partial e_{2}}\left[\alpha_{1} u_{I}-\alpha_{2} x_{2}-\alpha_{3}\right. & \\
& \left.-T_{f n}\left(x_{2}, \sigma_{0} z, \beta\right)+\Delta-\dot{x}_{2 e q}\right] .
\end{aligned}
$$


The (25) and (26) imply that, for any $\left|e_{\varepsilon}\right|>0$, we have $e_{\varepsilon} / e_{2}>$ 0 . Hence, to make $e_{\varepsilon}$ converge to zero, we only need to let $\alpha_{1} u_{I}-\alpha_{2} x_{2}-\alpha_{3}-T_{f n}\left(x_{2}, \sigma_{0} z, \beta\right)+\Delta-\dot{x}_{2 e q}<0$. Similar to (14), it can be deduced that

$$
\begin{aligned}
& -e_{\varepsilon} T_{f n}\left(x_{2}, \sigma_{0} z, \beta\right) \\
& \quad \leq\left|e_{\varepsilon}\right| T_{\max } L^{T}\left(x_{2}\right)\left(\beta-\beta_{\min }\right)-e_{\varepsilon} T_{f n}\left(x_{2}, \sigma_{0} z, \beta_{\min }\right) \\
& \quad \leq\left|e_{\varepsilon}\right| T_{\max } L^{T}\left(x_{2}\right) \gamma+\left|e_{\varepsilon}\right| T_{\max } f\left(x_{2}, \beta_{\min }\right) .
\end{aligned}
$$

From the definition of $e_{\varepsilon}$, it is easy to verify that

$$
\left|e_{\varepsilon}\right|=e_{\varepsilon} \sigma_{\varepsilon}\left(e_{2}\right)
$$

where $\sigma_{\varepsilon}\left(e_{2}\right)$ is a continuous function of $e_{2}$ and defined as

$$
\sigma_{\varepsilon}\left(e_{2}\right)= \begin{cases}1 & e_{2}>(\sqrt{3}-1) \varepsilon / 2 \\ 2 e_{2} /(\sqrt{3} \varepsilon-\varepsilon) & \left|e_{2}\right| \leq(\sqrt{3}-1) \varepsilon / 2 \\ -1 & e_{2}<(1-\sqrt{3}) \varepsilon / 2\end{cases}
$$

Combining (28) and (29) with (27), one has

$$
\begin{array}{r}
\dot{V}_{\varepsilon} \leq e_{\varepsilon} \frac{\partial e_{\varepsilon}}{\partial e_{2}}\left[\alpha_{1} u_{I}-\alpha_{2} x_{2}-\alpha_{3}+\sigma_{\varepsilon}\left(e_{2}\right) T_{\max } L^{T}\left(x_{2}\right) \gamma\right. \\
\left.+\sigma_{\varepsilon}\left(e_{2}\right) T_{\max } f\left(x_{2}, \beta_{\min }\right)+\Delta-\dot{x}_{2 e q}\right]
\end{array}
$$

Based on the above inequality, the continuous control law can be synthesized as

$$
\left\{\begin{array}{c}
u_{I}=u_{a}+u_{s} \\
u_{a}=\frac{1}{\hat{\alpha}_{1}}\left[\hat{\alpha}_{2} x_{2}+\hat{\alpha}_{3}-\sigma_{\varepsilon}\left(e_{2}\right) T_{\max } L^{T}\left(x_{2}\right) \hat{\gamma}+\dot{x}_{2 e q}\right. \\
\left.\quad \quad-\sigma_{\varepsilon}\left(e_{2}\right) T_{\max } f\left(x_{2}, \beta_{\min }\right)\right] \\
u_{s}=u_{s 1}+u_{s 2}, u_{s 1}=-\frac{k_{s 1}}{\alpha_{1 \min }} e_{\varepsilon}
\end{array}\right.
$$

The robust control function $u_{s 2}$ should be selected to satisfy

$$
\begin{aligned}
e_{\varepsilon} u_{s 2} & \leq 0 \\
e_{\varepsilon}\left[\alpha_{1} u_{s 2}-\varphi^{T} \tilde{\theta}+\Delta\right] & \leq \varepsilon_{1}
\end{aligned}
$$

where $\varphi^{T}=\left[u_{a},-x_{2},-1, \sigma_{\varepsilon}\left(e_{2}\right) T_{\max } L^{T}\left(x_{2}\right)\right]^{T}$, and $\varepsilon_{1}>0$ is a design parameter which can be arbitrarily small. Similar to the discontinuous control law design, one alternative of $u_{s 2}$ is

$$
u_{s 2}=-h /\left(2 \alpha_{1 \min } \varepsilon_{1} e_{\varepsilon}\right)
$$

where $h$ is any function or constant satisfying $h \geq\|\varphi\|^{2} \| \theta_{\max }-$ $\theta_{\text {min }} \|^{2}+\delta^{2}$. Thus, the continuous control law can assure the closed-loop system stable if the adaption law (20) is applied with $\tau=e_{\varepsilon}\left(\partial e_{\varepsilon} / \partial e_{2}\right) \varphi$. The rigorous proof of this result will be given in the Section IV. It will be verified that, by utilizing the above adaptation law, the modified tracking-error-index-like variable $e_{\varepsilon}$ can be steered to origin, in the presence of parametric uncertainties only.

\section{Stability Proof and Performance Analysis}

To analyze the control performance, we define the following level set of the positive definite function $V_{\varepsilon}$, for any $\rho>0$

$$
L_{V_{\varepsilon}}(\rho)=\left\{e_{\varepsilon}: V_{\varepsilon}\left(e_{\varepsilon}\right)=(1 / 2) e_{\varepsilon}^{2} \leq \rho\right\}
$$

In addition, a constant $\varepsilon^{\prime}$ is defined as

$$
\varepsilon^{\prime}=v_{0}+\max \left((2-\sqrt{3}) \varepsilon^{2} / 3, \quad \varepsilon_{1} / \lambda\right)
$$

where $v_{0}$ is any positive number. It can be arbitrarily small.

Lemma 4: Suppose that the control law (31) and the adaptation law (20) are applied to control the plant (6), which satisfies Assumptions $1 \sim 4$. Then, no matter what the adaptation function $\tau$ is, the controller guarantees the closed-loop system to be globally stable, and the trajectories starting outside $L_{V_{\varepsilon}}\left(\varepsilon^{\prime}\right)$ will enter $L_{V_{\varepsilon}}\left(\varepsilon^{\prime}\right)$ in a finite time and remain inside $L_{V_{\varepsilon}}\left(\varepsilon^{\prime}\right)$ thereafter; moreover, the trajectories starting from $L_{V_{\varepsilon}}\left(\varepsilon^{\prime}\right)$ will remain inside this level set.

Proof: See Appendix B.

Remark 3: From the proof of Lemma 4, we observe that, the tracking-error-index-like variable $e_{\varepsilon}$ can be steered into a sufficiently small region by increasing the robust control gain. However, every practical system is subject to input saturation. To avoid too large control action, the robust control gain should not be set too large. In fact, the adaptive control term can further improve the accuracy of the servo system, which will be demonstrated in the following deduction.

Theorem 1: Under the assumptions $1 \sim 4$, the closed-loop system comprised of the plant (6), the control law (31) and the adaptation law (20) has the following properties.

1) No matter what the adaptation function $\tau$ is, the trackingerror-index-like variable $e_{2}$ satisfies $\left|e_{2}(t)\right| \leq \sqrt{2 \varepsilon^{\prime}}+\varepsilon$, $\forall t \geq t_{0}, \exists t_{0}>0$, meaning that the tracking error will be within a prescribed bound after a finite time.

2) If $\Delta=0$, i.e., in the presence of parametric uncertainties only, and the adaptation function $\tau$ is given by (20) with $\tau=e_{\varepsilon}\left(\partial e_{\varepsilon} / \partial e_{2}\right) \varphi$, then, in addition to results in i), we have $e_{\varepsilon} \rightarrow 0$ as $t \rightarrow \infty$, which implies that $\left|e_{2}(t)\right| \leq$ $(\sqrt{3}-1) \varepsilon / 2$ as $t \rightarrow \infty$.

Proof: See Appendix C.

Remark 4: Theorem 1 shows that the tracking error bound of the closed-loop system with the adaptation function (20) is much smaller than that of the system without considering the adaptation law. Thus, the tracking accuracy is improved by parameter adaptation. On the other hand, due to the utilization of parameter adaptation, the system can achieve high accuracy without using large robust control gain.

\section{EXPERIMENTAL RESULTS}

In this section, experimental results are obtained from a DC torque motor servomechanism to demonstrate the effectiveness of the proposed method.

\section{A. Experimental Setup}

According to our previous work [27], a two-axis turntable servo system (see Fig. 1) is set up as a test-bed.

The position and velocity signals are collected by the optical encoder with 0.0005 degree resolution. The controller is implemented through an Xpc-Target that consists of a target personal computer and the interface card NI PCI-6052E. The sampling rate of the servo controller is $2 \mathrm{kHz}$. Experiments are con- 


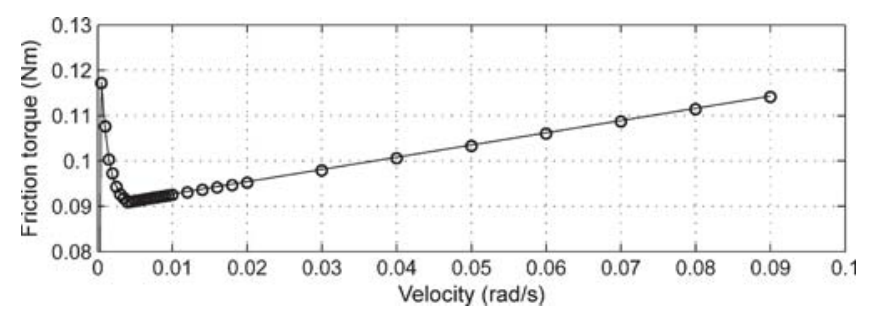

Fig. 2. Stribeck curve.

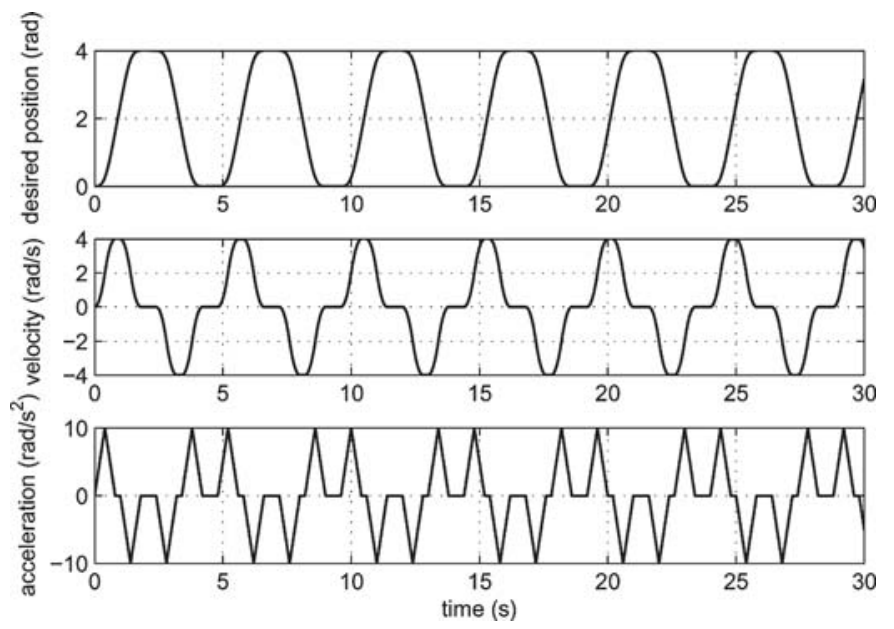

Fig. 3. Desired trajectory.

ducted on the pitch-axis which undertakes the payload (e.g., TV camera). The unbalance payload is considered as the external disturbance.

\section{B. Determination of the Bounds of Unknown Parameters}

From the (6), we know that the unknown parameters $\alpha_{i}, \beta_{j}$ are relative to $K_{I}, J$, average load torque $T_{n}$ and parameters in friction model. From our previous work [27], we know $J=0.011 \mathrm{kgm}^{2}, K_{I}=6.36 \mathrm{~N} / \mathrm{A}, T_{n}=0.1 \mathrm{Nm}$. To determine the friction parameters, offline parameter identification is carried out following the same procedure as in [10]. The Stribeck curve is shown in Fig. 2, from which we can roughly get $g\left(x_{2}\right)=0.09171+0.0255 e^{-(x / 0.0477)^{2}} \mathrm{Nm}$ and $\sigma_{2}=0.2702 \mathrm{Nm} /(\mathrm{rad} / \mathrm{s})$.

To obtain $\sigma_{0}, \sigma_{1}$, let the system operate around zero velocity, give it a step input and measure the output response. With these experiments, finally, we get $\sigma_{0}=421.6$ $\mathrm{N}$ and $\sigma_{1}=6.738 \mathrm{Nm} /(\mathrm{rad} / \mathrm{s})$. Consequently, we can get $T_{c}=0.09171 \mathrm{~N}, T_{s}=T_{c}+0.0255=0.11721 \mathrm{~N}$. The motor driver is set as torque mode. The gain of the pseudo amplifier is $k_{a}=2.4$ obtained from the driver specification. Then noting (6), we know that $\alpha_{1}=K_{I} k_{a} / J=1387.63(\mathrm{~N} / \mathrm{A}) /\left(\mathrm{kgm}^{2}\right)$, $\alpha_{2}=\left(\sigma_{1}+\sigma_{2}\right) / J=637.12 \mathrm{rad}^{-1} \mathrm{~s}^{-1}, \alpha_{3}=T_{n} / J=$ $9.09 \mathrm{~N} /\left(\mathrm{kgm}^{2}\right), \beta_{1}=1 / J=90.91\left(\mathrm{kgm}^{2}\right)^{-1}, \beta_{2}=$ $\sigma_{1} / T_{c}=61.25 \mathrm{~m} /(\mathrm{rad} / \mathrm{s}), \beta_{3}=T_{s} / T_{c}=1.278$, $\beta_{4}=1 / \omega_{s}^{2}=500\left[(\operatorname{rad} / \mathrm{s})^{2}\right]^{-1}$. The bounds of the parameter variations in the experiment are chosen as $\alpha_{\min }=[1248,573,8]^{T}, \alpha_{\max }=[1526,701,10]^{T}$, $\beta_{\min }=[81,55,1.1,450]^{T}, \beta_{\max }=[100,68,1.5,550]^{T}$. To

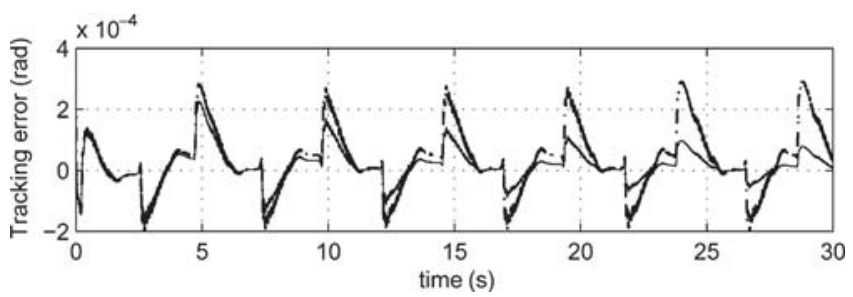

Fig. 4. Tracking performance (solid line: proposed method, dashed-dotted line: deterministic robust controller).

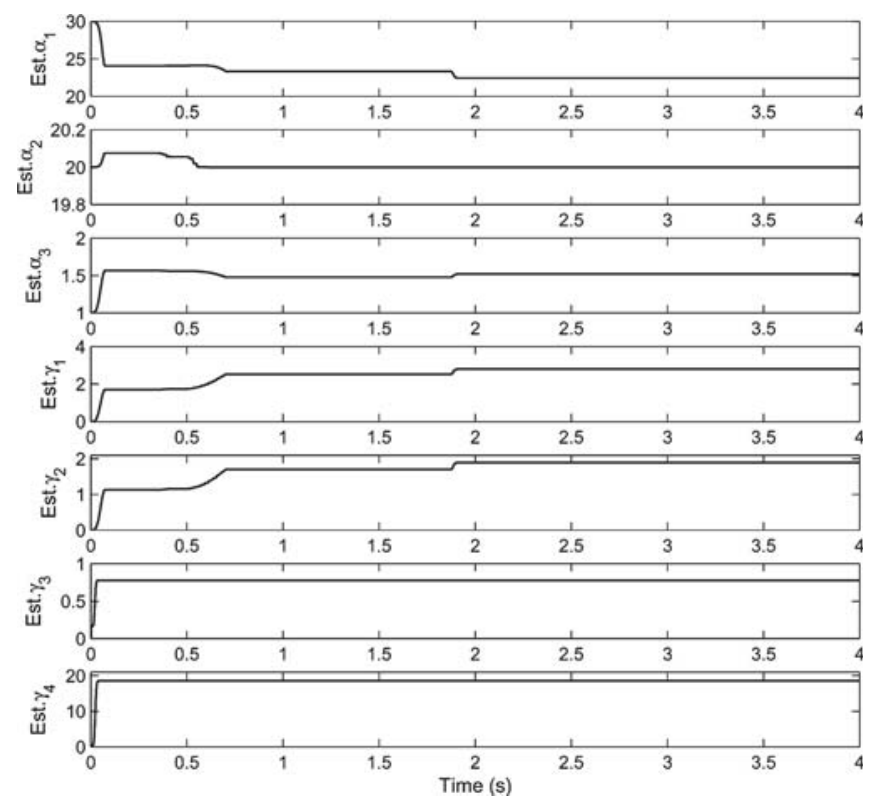

Fig. 5. Parameter estimates.

determined the value of $T_{\max }$, the pitch-axis is forced to run at a constant velocity. Then from (4), one has $\sigma_{0} z=g\left(x_{2}\right) \operatorname{sgn}\left(x_{2}\right)$. Consequently, the value of $T_{\max }$ is estimated as $T_{\max }=0.2$ $\mathrm{Nm}$.

From the definition of control robust term $u_{s 2}$, one knows that the control gain is determined by the bounds of unknown parameters. It indicates that if the bounds are chosen too loosely, the final controller may be very conservative. Even more, in the real application, it will lead the controller to become saturated and make the system unstable. As a result, we first estimate the unknown parameters roughly in the system through offline identification. Then the lower bound and upper bound are set as less or more than $10 \%$ of the estimates, respectively [28], [29]. Actually, in real application, the feedback gain of the robust controller term is easily determined by try-and-error method.

\section{Experimental Results}

In our experiment, the unbalance torque caused by gravitational torque is considered as the main external disturbance. The constant offset is estimated online. Thus, the value of $\delta$ is supposed as $\delta \leq 0.05$. The controller parameters are chosen as follows:

$$
\begin{aligned}
& k_{p}=5, k_{s 1}=5, u_{s 2}=-k_{s 2} e_{\varepsilon}, k_{s 2}=10 \\
& \Gamma=\operatorname{diag}(800,20,50,50,10,10,1 e 4), \varepsilon=0.001, b=2 .
\end{aligned}
$$


Noting the definition of $u_{s 2}$, one has $k_{s 2}=h /\left(2 \alpha_{1 \min } \varepsilon_{1}\right)$. In the real application, the value of $k_{s 2}$ is chosen by try-anderror method. The desired trajectory is shown in Fig. 3 (i.e., with maximum velocity $\pm 4 \mathrm{rad} / \mathrm{s}$ and maximum acceleration of $\pm 10 \mathrm{rad} / \mathrm{s}^{2}$ ). The tracking performance and control effort are shown in Fig. 4 and Fig. 5. (The curves of parameter estimates and error $e_{2}$ are omitted due to the limited space.)

From Fig. 4, we can see that the tracking error decreases gradually because of the parameter adaptation. As seen from the Fig. 5, the control effort varying between $+5 \mathrm{~V}$ and $-5 \mathrm{~V}$ is up to half of the limits. This indicates that a more accurate tracking error could be obtained if the control gain increases.

\section{CONCLUSION}

We have developed an adaptive robust control (ARC) scheme with dynamic friction compensation for a DC servomechanism. This scheme utilizes a Lipschitzian property to re-parameterize the plant, and thus the number of parameters to be updated in the $\mathrm{ARC}$ is equal to the number of unknown parameters of the plant. Moreover, the friction state observer is not needed in this method. Thus, the resulting control algorithm is convenient for engineering application. It has been proved that the proposed method can not only guarantee desired transient performance for the system, but also make the magnitude of steady-state tracking error arbitrarily small in the presence of parametric uncertainties only. The experimental results show that the proposed ARC scheme can achieve desirable control performance for the servo mechanisms with dynamic friction effects.

\section{APPENDIX A}

Proof: Let $\beta^{\prime} \in \Omega_{\beta}$ and $\beta^{\prime \prime} \in \Omega_{\beta}$ be fixed. Define $\eta(\rho)=$ $(1-\rho) \beta^{\prime}+\rho \beta \prime \prime$ for all $\rho \in[0,1]$. It is easy to verify that $\eta(\rho) \in \Omega_{\beta}$. Since, in the domain $R \times \Omega_{\beta}$, the function $f\left(x_{2}, \beta\right)$ is continuously differentiable in $\beta$ for any fixed $x_{2} \in R$, we can conclude that $f\left(x_{2}, \eta(\rho)\right)$ is also continuously differentiable in $\rho$. According to the mean value theorem, there exists $\rho_{1} \in[0,1]$ such that

$$
\begin{aligned}
f\left(x_{2}, \beta^{\prime \prime}\right)-f\left(x_{2}, \beta^{\prime}\right) & =\left.\frac{\partial f\left(x_{2}, \beta\right)}{\partial \beta} \frac{\partial \beta}{\partial \rho}\right|_{\rho=\rho_{1}} \\
& =\frac{\partial f}{\partial \beta}\left(x_{2}, \eta\left(\rho_{1}\right)\right)\left(\beta^{\prime \prime}-\beta^{\prime}\right) .
\end{aligned}
$$

Since $\eta\left(\rho_{1}\right) \in \Omega_{\beta}$, we have

$$
\begin{aligned}
& \left|f\left(x_{2}, \beta^{\prime}\right)-f\left(x_{2}, \beta^{\prime \prime}\right)\right| \\
& \quad \leq \sum_{i=1}^{4}\left|\beta_{i}^{\prime}-\beta_{i}^{\prime \prime}\right| \max _{0<\rho<1}\left|\frac{\partial f}{\partial \beta_{i}}\left(x_{2}, \eta(\rho)\right)\right| \\
& \quad \leq \sum_{i=1}^{4}\left|\beta_{i}^{\prime}-\beta_{i}^{\prime \prime}\right| \max _{\beta \in \Omega_{\beta}}\left|\frac{\partial f}{\partial \beta_{i}}\left(x_{2}, \beta\right) .\right|
\end{aligned}
$$

From Definition 1 and the above inequality, we can obtain the result of Lemma 2.

\section{APPENDIX B}

Proof: The proof of Lemma 4 is divided into two parts, i.e., i) $e_{\varepsilon} \in R \backslash L_{V_{\varepsilon}}\left(\varepsilon^{\prime}\right)$; ii) $e_{\varepsilon} \in L_{V_{\varepsilon}}\left(\varepsilon^{\prime}\right)$.

i) Consider the case of $e_{\varepsilon} \in R \backslash L_{V_{\varepsilon}}\left(\varepsilon^{\prime}\right)$. According to (34), the states outside $L_{V_{\varepsilon}}\left(\varepsilon^{\prime}\right)$ satisfy $V_{\varepsilon}\left(e_{\varepsilon}\right)>\varepsilon^{\prime}$, which implies $\left|e_{\varepsilon}\right|>(\sqrt{3}-1) \varepsilon / \sqrt{3}$. From the definition of $e_{\varepsilon}$, it can be derived that $\left|e_{2}\right|>\varepsilon$. Then, according to (26), we obtain that $\partial e_{\varepsilon} / \partial e_{2}=1$. Substituting (31) into (30), we have

$$
\begin{aligned}
& \dot{V}_{\varepsilon} \leq e_{\varepsilon} \frac{\partial e_{\varepsilon}}{\partial e_{2}}[-k_{s 1} e_{\varepsilon}+\alpha_{1}\left(u_{a}+u_{s 2}\right)-\alpha_{2} x_{2}-\alpha_{3} \\
&+\sigma_{\varepsilon}\left(e_{2}\right) T_{\max }\left(L^{T}\left(x_{2}\right) \gamma+f\left(x_{2}, \beta_{\min }\right)\right) \\
&\left.\dot{x}_{2 e q}+\Delta\right] \\
&=-k_{s 1} e_{\varepsilon}^{2}+e_{\varepsilon}\left[\alpha_{1} u_{s 2}-\tilde{\alpha}_{1} u_{a}+\tilde{\alpha}_{2} x_{2}+\tilde{\alpha}_{3}\right. \\
&\left.\quad-\sigma_{\varepsilon}\left(e_{2}\right) T_{\max } L^{T}\left(x_{2}\right) \tilde{\gamma}+\Delta\right] \\
& \forall e_{\varepsilon} \in R \backslash L_{V_{\varepsilon}}\left(\varepsilon^{\prime}\right) .
\end{aligned}
$$

Combining with (33) and (35), it follows that

$$
\dot{V}_{\varepsilon} \leq-\lambda V_{\varepsilon}+\varepsilon_{1}<-\lambda \varepsilon^{\prime}+\varepsilon_{1} \leq-\lambda v_{0} \quad \forall e_{\varepsilon} \in R \backslash L_{V_{\varepsilon}}\left(\varepsilon^{\prime}\right)
$$

Thus, it can be concluded that the trajectories starting outside $L_{V_{\varepsilon}}\left(\varepsilon^{\prime}\right)$ will enter $L_{V_{\varepsilon}}\left(\varepsilon^{\prime}\right)$ in a finite time.

ii) Consider the case of $e_{\varepsilon} \in L_{V_{\varepsilon}}\left(\varepsilon^{\prime}\right)$. In this case, we have

$$
\begin{aligned}
& \dot{V}_{\varepsilon} \leq e_{\varepsilon} \frac{\partial e_{\varepsilon}}{\partial e_{2}}[- k_{s 1} e_{\varepsilon}+\alpha_{1}\left(u_{a}+u_{s 2}\right)-\alpha_{2} x_{2}-\alpha_{3} \\
&+\sigma_{\varepsilon}\left(e_{2}\right) T_{\max }\left(L^{T}\left(x_{2}\right) \gamma+f\left(x_{2}, \beta_{\min }\right)\right) \\
&\left.-\dot{x}_{2 e q}+\Delta\right] \\
& \leq \frac{\partial e_{\varepsilon}}{\partial e_{2}}\left(-\lambda V_{\varepsilon}+\varepsilon_{1}\right) \quad \forall e_{\varepsilon} \in L_{V_{\varepsilon}}\left(\varepsilon^{\prime}\right)
\end{aligned}
$$

From (26), one has $\partial e_{\varepsilon} / \partial e_{2} \geq 0$. Then, noting that $\varepsilon^{\prime}>$ $\varepsilon_{1} / \lambda$, in view of (38), it follows that

$$
\dot{V}_{\varepsilon} \leq 0 \quad \forall e_{\varepsilon} \in L_{V_{\varepsilon}}\left(\varepsilon^{\prime}\right) \backslash L_{V_{\varepsilon}}\left(\varepsilon_{1} / \lambda\right)
$$

which implies that when $e_{\varepsilon} \in L_{V_{\varepsilon}}\left(\varepsilon^{\prime}\right) \backslash L_{V_{\varepsilon}}\left(\varepsilon_{1} / \lambda\right)$, the Lyapunov function $V_{\varepsilon}$ must be decreasing. Thus, the states inside $L_{V_{\varepsilon}}\left(\varepsilon^{\prime}\right)$ will not escape $L_{V_{\varepsilon}}\left(\varepsilon^{\prime}\right)$ and remain in it thereafter.

Combining the analysis in i) and ii), we can obtain the results in Lemma 4.

\section{APPENDIX C}

Proof: From Lemma 4, we know that, no matter what the initial conditions are, $e_{\varepsilon}$ will enter the level set $L_{V_{\varepsilon}}\left(\varepsilon^{\prime}\right)$ after a finite time of running. It implies that $\left|e_{\varepsilon}\right| \leq \sqrt{2 \varepsilon^{\prime}}, \forall t \geq t_{0}, \exists t_{0}>$ 0 . From the definition of $e_{\varepsilon}$, we have $e_{2}=e_{\varepsilon}+c\left(e_{2}\right)$. Since $\left|c\left(e_{2}\right)\right| \leq \varepsilon$, it can be concluded that $\left|e_{2}(t)\right| \leq \sqrt{2 \varepsilon^{\prime}}+\varepsilon$, $\forall t \geq t_{0}, \exists t_{0}>0$, which completes the proof of i).

Then, we prove ii). Define a positive definite function $V_{\theta}$ as

$$
V_{\theta}=\frac{1}{2} e_{\varepsilon}^{2}+\frac{1}{2} \tilde{\theta}^{T} \Gamma^{-1} \tilde{\theta}
$$


If $\Delta=0$, then from (6), (20), (31) and (34), the derivative of $V_{\theta}$ can be derived as

$$
\begin{aligned}
\dot{V}_{\theta} \leq & \frac{\partial e_{\varepsilon}}{\partial e_{2}} e_{\varepsilon}\left(-k_{s 1} e_{\varepsilon}-\varphi^{T} \tilde{\theta}+\alpha_{1} u_{s 2}\right) \\
& +\tilde{\theta}^{T} \Gamma^{-1} \operatorname{Proj}_{\hat{\theta}}\left(\Gamma \frac{\partial e_{\varepsilon}}{\partial e_{2}} e_{\varepsilon} \varphi\right) \\
= & -\frac{\partial e_{\varepsilon}}{\partial e_{2}} k_{s 1} e_{\varepsilon}^{2}+\frac{\partial e_{\varepsilon}}{\partial e_{2}} \alpha_{1} u_{s 2} e_{\varepsilon} \\
& +\tilde{\theta}^{T} \Gamma^{-1}\left[\operatorname{Proj}_{\hat{\theta}}\left(\Gamma \frac{\partial e_{\varepsilon}}{\partial e_{2}} e_{\varepsilon} \varphi\right)-\frac{\partial e_{\varepsilon}}{\partial e_{2}} e_{\varepsilon} \varphi\right] .
\end{aligned}
$$

Noting that $\left(\partial e_{\varepsilon} / \partial e_{2}\right) \geq 0$ and $\alpha_{1} \geq a_{1 \text { min }}>0$, According to the properties of projection operation and (32), the above inequalities can be simplified as

$$
\dot{V}_{\theta} \leq-\frac{\partial e_{\varepsilon}}{\partial e_{2}} k_{s 1} e_{\varepsilon}^{2}
$$

Define a quantity $f_{\varepsilon}$ as $f_{\varepsilon}:=k_{s 1} e_{\varepsilon}^{2} \partial e_{\varepsilon} / \partial e_{2}$. Then, integrating two sides of (41) from $t=0$ to $t=\infty$ yields

$$
\lim _{t \rightarrow \infty} \int_{0}^{t} f_{\varepsilon}(\tau) d \tau \leq V_{\theta}(0)-V_{\theta}(\infty) .
$$

Noting that $(1 / 2) \tilde{\theta}^{T} \Gamma^{-1} \tilde{\theta}$ is always bounded due to the projection mapping, from the result of Lemma 4, we know that $V_{\theta}(\infty)$ is bounded. Accordingly, it can be concluded that $\lim _{t \rightarrow \infty} \int_{0}^{T} f_{\varepsilon}(\tau) d \tau$ exists and is finite.

On the other hand, we can also derive that $f_{\varepsilon}$ is uniformly continuous. The deduction is as follows. The definition of $f_{\varepsilon}$ shows that $f_{\varepsilon}=2 k_{s 1}\left(\partial e_{\varepsilon} / \partial e_{2}\right) V_{\varepsilon}$. Noting that $V_{\varepsilon}$ is bounded, which can be justified by Lemma 4 , we denote the upper bound of $V_{\varepsilon}$ as $B_{V}$, i.e., $V_{\varepsilon} \leq B_{V}$. Besides, the (26) shows that $\left|\partial e_{\varepsilon} / \partial e_{2}\right| \leq 1$. From (36), (38) and Lemma 4, we know that $\dot{V}_{\varepsilon}$ is bounded, thus $V_{\varepsilon}$ is uniformly continuous, namely, for any $t_{1}, t \geq 0$ and $R_{1}>0$, there exists $\eta_{1}\left(R_{1}\right)>0$ such that $\left|t-t_{1}\right|<\eta_{1}$ implies $\left|V_{\varepsilon}(t)-V_{\varepsilon}\left(t_{1}\right)\right|<R_{1}$. On the other hand, from the definition of $\partial e_{\varepsilon} / \partial e_{2}$, we can observe that $\partial e_{\varepsilon} / \partial e_{2}$ is also uniformly continuous, and thus for any $R_{2}>0$, there exist $\eta_{2}\left(R_{2}\right)>0$ such that $\left|t-t_{1}\right|<\eta_{2}$ implies $\left|\left(\partial e_{\varepsilon} / \partial e_{2}\right)(t)-\left(\partial e_{\varepsilon} / \partial e_{2}\right)\left(t_{1}\right)\right|<R_{2}$. For any $R>0$, we select $R_{1}$ and $R_{2}$ such that $2 k_{s 1}\left(R_{1}+B_{V} R_{2}\right) \leq R$. Then, it can be obtained that, if $\left|t-t_{1}\right|<\eta:=\min \left(\eta_{1}, \eta_{2}\right)$, then

$$
\begin{aligned}
\left|f_{\varepsilon}\left(t_{1}\right)-f_{\varepsilon}(t)\right| & =2 k_{s 1}\left|\frac{\partial e_{\varepsilon}}{\partial e_{2}}\left(t_{1}\right) V_{\varepsilon}\left(t_{1}\right)-\frac{\partial e_{\varepsilon}}{\partial e_{2}}(t) V_{\varepsilon}(t)\right| \\
\leq 2 k_{s 1}\left(\left|V_{\varepsilon}\left(t_{1}\right)\right|\left|\frac{\partial e_{\varepsilon}}{\partial e_{2}}\left(t_{1}\right)-\frac{\partial e_{\varepsilon}}{\partial e_{2}}(t)\right|\right. & \left.\quad+\left|\frac{\partial e_{\varepsilon}}{\partial e_{2}}(t)\right|\left|V_{\varepsilon}\left(t_{1}\right)-V_{\varepsilon}(t)\right|\right) \\
& <2 k_{s 1}\left(R_{1}+B_{V} R_{2}\right) \leq R .
\end{aligned}
$$

Since $R$ is arbitrarily chosen, $f_{\varepsilon}$ is uniformly continuous.

Therefore, it can be concluded by the Barbatlat's lemma that $f_{\varepsilon} \rightarrow 0$ as $t \rightarrow \infty$. From (26) and the definition of $e_{\varepsilon}$, one has $\partial e_{\varepsilon} / \partial e_{2}=0$ if and only if $e_{\varepsilon}=0$, and then we obtain $f_{\varepsilon} \rightarrow 0$ equals to $e_{\varepsilon} \rightarrow 0$. In view of the definition of $e_{\varepsilon}$, it follows that $\left|e_{2}(t)\right| \leq(\sqrt{3}-1) \varepsilon / 2$ as $t \rightarrow \infty$, which completes the proof of result ii).

\section{REFERENCES}

[1] B. Armstrong-Helouvry, P. Dupont, and C. Canudas de Wit, "A survey of analysis tools and compensation methods for the control of machines with friction," Automatica, vol. 30, no. 7, pp. 1083-1138, 1994.

[2] B. Bona and M. Indri, "Friction compensation in robotics: An overview," in Proc. 44th IEEE Conf. Decision Control, 2005, pp. 4360-4367.

[3] D. Kostic, B. de Jager, M. Steinbuch, and R. Hensen, "Modeling and identification for high-performance robot control: An RRR-robotic arm case study," IEEE Trans. Control Syst. Technol., vol. 12, no. 6, pp. 904-919, Nov. 2004.

[4] J. Moreno, R. Kelly, and R. Campa, "Manipulator velocity control using friction compensation," IEE Proc.-Control Theory Appl., vol. 150, no. 2, pp. 119-126, 2003.

[5] D. Garagic and K. Srinivasan, "Adaptive friction compensation for precision machine tool drive," Control Eng. Practice, vol. 12, no. 11, pp. 1451-1464, 2004.

[6] S. S. Ge, T. H. Lee, and S. X. Ren, "Adaptive friction compensation of servo mechanisms," Int. J. Syst. Sci., vol. 32, no. 4, pp. 523-532, 2001.

[7] I. Nilkhamhang and A. Sano, "Model-based adaptive friction compensation for accurate position control," in Proc. 47th IEEE Conf. Decision Control, 2008, pp. 1346-1351.

[8] H. Olsson, K. J. Astrom, C. Canudas de Wit, M. Gafvert, and P. Lischinsky, "Friction models and friction compensation," Euro. J. Cont., vol. 4, pp. 176-195, 1998.

[9] P. Dahl, "A solid friction model," The Aerospace Corporation, El Segundo, CA, Tech. Rep. TOR-158(3107-18), 1968.

[10] C. Canudas de Wit, H. Olsson, K. J. Astrom, and P. Lischinsky, “A new model for control of systems with friction," IEEE Trans. Autom. Control, vol. 40, no. 3, pp. 419-425, 1995.

[11] C. Canudas de Wit and P. Lischinsky, "Adaptive friction compensation with partially known dynamic friction model," Int. J. Adapt. Cont. Signal Proc., vol. 11, no. 1, pp. 65-80, 1997.

[12] P. Vedagarbha, J. M. Dawson, and M. Feemster, "Tracking control of mechanical systems in the presence of nonlinear dynamic friction effects," IEEE Trans. Control Syst. Technol., vol. 7, no. 4, pp. 446-456, 1999.

[13] T. H. Lee, K. K. Tan, and S. Huang, "Adaptive friction compensation with a dynamical friction model," IEEE/ASME Trans. Mechatron. 10.1109/TMECH.2009.2036994.

[14] S. S. Ge, T. H. Lee, and J. Wang, "Adaptive NN control of dynamic systems with unknown dynamic friction," in Proc. 39th IEEE Conf. Decision Control, 2000, pp. 1760-1765.

[15] L. Xu and B. Yao, "Adaptive robust control of mechanical systems with non-linear dynamic friction compensation," Int. J. Control, vol. 81, no. 2, pp. 167-176, 2008.

[16] B. Yao and M. Tomizuka, "Adaptive robust control of SISO non-linear systems in a semi-strict feedback form," Automatica, vol. 33, no. 5, pp. 893-900, 1997.

[17] X. Liu, H. Su, B. Yao, and J. Chu, "Adaptive robust control of nonlinear systems with dynamic uncertainties," Int. J. Adapt. Control Signal Process., vol. 23, no. 4, pp. 353-377, 2009.

[18] L. Lu, B. Yao, Q. Wang, and Z. Chen, "Adaptive robust control of linear motors with dynamic friction compensation using modified LuGre model," Automatica, vol. 45, no. 12, pp. 2890-2896, 2009.

[19] N. V. Q. Hung, H. D. Tuan, T. Narikiyo, and P. Apkarian, "Adaptive control for nonlinearly parameterized uncertainties in robot manipulators," IEEE Trans. Control Syst. Technol., vol. 16, no. 3, pp. 458-468, May 2008.

[20] K. Yokoi, N. V. Q. Hung, H. D. Tuan, and S. Hosoe, "Adaptive control design for nonlinearly parameterized systems with a triangular structure," Asian J. Control, vol. 9, no. 2, pp. 121-132, 2007.

[21] K. K. Tan and S. N. Huang et al., "7Adaptive robust motion control for precise trajectory tracking applications," ISA Trans., vol. 40, no. 1, pp. 57-71, 2001.

[22] J. Wang, S. S. Ge, and T. H. Lee, "Adaptive friction compensation for servo mechanisms," in Adaptive Control of Nonsmooth Dynamic Systems, G. Tao and F. L. Lewis, Eds. Berlin, Germany: Springer, 2001, pp. 211-248.

[23] G. C. Goodwin and D. Q. Mayne, “A parameter estimation perspective of continuous time model reference adaptive control," Automatica, vol. 23 , no. 1 , pp. 57-70, 1989. 
[24] B. Yao and M. Tomizuka, "Smooth robust adaptive sliding mode control of robot manipulators with guaranteed transient performance," $J$. Dyn. Syst., Meas. Control, vol. 18, no. 4, pp. 764-775, 1996.

[25] B. Yao, "High performance adaptive robust control of nonlinear systems: A general framework and new schemes," in Proc. IEEE Conf. Decision Control, 1997, pp. 2489-2494.

[26] L. Lu, Z. Chen, B. Yao, and Q. Wang, "Desired compensation adaptive robust control of a linear-motor-driven precision industrial gantry with improved cogging force compensation," IEEE/ASME Trans. Mechatron., vol. 13, no. 6, pp. 617-624, Dec. 2008.

[27] G. Zhang, J. Chen, and Z. Li, "Adaptive robust control for servo mechanisms with partially unknown states via dyanmic surface control approach," IEEE Trans. Control Syst. Technol., vol. 18, no. 3, pp. 723-731, May 2010.
[28] K. Worden, C. X. Wong, U. Parlitz, and A. Hornstein et al., "Identification of pre-sliding and sliding friction dynamics: Grey box and black-box models," Mechan. Syst. Signal Process., vol. 21, pp. 514-534, 2007.

[29] L. Xu and B. Yao, "Adaptive robust motion control of linear motors for precision manufacturing," Mechatronics, vol. 12, no. 4, pp. 595-616, 2002. 